\title{
Measurement of $e^{+} e^{-} \rightarrow \mu^{+} \mu^{-}:$A Test of Electroweak Theories
}

B. Adeva, U. Becker, R. Becker-Szendy, J. Berdugo, A. Boehm, J. G. Branson, J. D. Burger, M. Capell, M. Cerrada, C. C. Chang, Y. H. Chang, H. S. Chen, M. Chen, M. L. Chen, M. Y. Chen, Y. S. Chu, E. Deffur, K. Deiters, M. Demarteau, B. Z. Dong, P. Duinker, H. S. Fesefeldt, D. Fong, M. Fukushima, L. Garrido, R. D. Han, D. Harting, G. Herten, M. C. Ho, D. Hueser, M. Hussain, M. M. Ilyas, D. Z. Jiang, M. Klein, W. Krenz, P. Kuijer, R. Leiste, Q. Z. Li, D. Linnhoefer, D. Luckey, E. J. Luit, C. Mana, M. A. Marquina, M. Martinez, G. G. G. Massaro, J. Mnich, R. Mount, K. Nadeem, H. Newman, W. D. Nowak, M. Pohl, F. P. Poschmann, R. R. Rau, S. Rodriguez, M. Rohde, J. A. Rubio, H. Rykaczewski, M. Sachwitz, J. Salicio, H. J. Schreiber, U. Schroeder, J. Schug, H. Stone, G. M. Swider, H. W. Tang, D. Teuchert, Samuel C. C. Ting, K. L. Tung, H. Vogt, M. Q. Wang, M. White, H. G. Wu, S. X. Wu, B. Wyslouch, B. X. Yang, B. Zhou, and R. Y. Zhu

(The Mark J Collaboration)

III. Physikalisches Institut, Technische Hochschule, D-5100 Aachen, Federal Republic of Germany
Brookhaven National Laboratory, Upton, New York 11973
California Institute of Technology, Pasadena, California 91125
Deutsches Elektronen Synchrotron DESY, D-2000 Hamburg 52, Federal Republic of Germany
Laboratory for Nuclear Science, Massachusetts Institute of Technology, Cambridge, Massachusetts 02139
Junta de Energia Nuclear, Madrid, Spain
Nationaal Instituut voor Kernfysica en Hoge-Energiefysica, 1009-DB Amsterdam, The Netherlands
Institute of High Energy Physics, Chinese Academy of Science, Peking, People's Republic of China
Institut für Hochenergiephysik der Angewandte der Wissenschaften der Deutsche Demokratische Republik,
1615 Berlin-Zeuthen, Deutsche Demokratische Republik
(Received 30 May 1985)

We use the reaction $e^{+} e^{-} \rightarrow \mu^{+} \mu^{-}$, in the Mark J detector at the DESY high-energy $e^{+} e^{-}$collider PETRA, to test the standard electroweak theory and find good agreement. We also set limits on the parameters of several extended gauge theories.

PACS numbers: $13.10 .+\mathrm{q}, 12.10 . \mathrm{Ck}$

The leptonic reaction

$$
e^{+} e^{-} \rightarrow \mu^{+} \mu^{-}
$$

is of particular importance for testing electroweak theories, since it is a pure $s$-channel reaction and the final-state particles can be well measured. The measurement of the interference effects between the electromagnetic and weak interactions at high energies determines the strength of the weak-neutral-current interaction. The (minimal) standard model is a $\mathrm{SU}(2)_{L} \otimes \mathrm{U}(1)$ gauge theory and in this interaction has two parameters, $\sin ^{2} \theta_{\mathrm{W}}\left(\theta_{\mathrm{W}}\right.$ is the weak mixing angle), and $m_{Z^{0}}$, the mass of the neutral vector boson $Z^{0}$. We use the measurement of reaction (1) as a test of the standard theory ${ }^{1}$ and we set limits on the parameters of extended gauge theories.

The Mark J detector has collected 5500 events of reaction (1) over a range of center-of-mass energies, $14 \mathrm{GeV}<\sqrt{s}<46.78 \mathrm{GeV}$. The largest sample $(3658$ events) was obtained with $\langle\sqrt{s}\rangle=34.6 \mathrm{GeV} .{ }^{2,3}$ (See Table I.) The measured angular distributions and details of event selection and a study of systematic effects have been discussed in Ref. 3. Other storagering experiments at PETRA $^{4}$ (DESY) and PEP $^{5}$ (SLAC) have published results on reaction (1) at somewhat lower energies.

The electroweak interaction predicts a deviation from the pointlike cross sections due to the $\gamma-Z^{0}$ interference, ${ }^{6}$

$$
R_{\mu \mu}=\sigma_{\mu \mu} / \sigma_{\text {point }}=1-2 g_{V}^{e} g_{V}^{\mu} \chi+\left[\left(g_{V}^{e}\right)^{2}+\left(g_{A}^{e}\right)^{2}\right]\left[\left(g_{V}^{\mu}\right)^{2}+\left(g_{A}^{\mu}\right)\right] \chi^{2},
$$

where $g_{V}, g_{A}$ are the vector and axial-vector couplings and

$$
\begin{aligned}
& \chi=\left(4 \sin ^{2} \theta_{\mathrm{W}} \cos ^{2} \theta_{\mathrm{W}}\right)^{-1}\left(s / m_{Z^{0}}^{2}-s\right), \\
& \sigma_{\text {point }}=4 \pi \alpha^{2} / 3 s,
\end{aligned}
$$

and $\alpha$ and $s$ have their usual meaning. In Eq. (3) we neglect the width of the $Z^{0}$ with respect to its mass. The vector and axial-vector couplings $g_{V}$ and $g_{A}$ are given in the $\mathrm{SU}(2)_{L} \otimes \mathrm{U}(1)$ model by

$$
g_{V}=-\frac{1}{2}+2 \sin ^{2} \theta_{\mathrm{W}}, \quad g_{A}=-\frac{1}{2} \text {. }
$$

Reaction (1) can be efficiently separated from the background reactions

$$
\begin{aligned}
& e^{+} e^{-} \rightarrow e^{+} e^{-} \mu^{+} \mu^{-}, \\
& e^{+} e^{-} \rightarrow \tau^{+} \tau^{-} \rightarrow \mu^{+} \mu^{-}+\text {neutrinos, }
\end{aligned}
$$


by selecting two muon events with an acollinearity angle smaller than $20^{\circ}$ and for which at least one muon has a momentum greater than half of the beam energy. The remaining background from each reaction in (6) is less than $1 \%$. We retain only events with $\cos \theta<0.8$ since the acceptance in this angular region is flat and above $90 \%$. ( $\theta$ is the angle between the outgoing $\mu^{-}$ and the incoming $e^{-}$.) The measured data are corrected for acceptance and for QED radiative effects ${ }^{7}$ to order $\alpha^{3}$. The flat acceptance and the good resolution of the Mark $\mathrm{J}$ detector lead to a $4 \%$ systematic error in the cross section, of which $3 \%$ is from the uncertainty in the luminosity. With $\sin ^{2} \theta_{\mathrm{W}}=0.22$, the standard model predicts that the weak vector couplings of the electron and muon are near zero, and thus at our energies $R_{\mu \mu}$ should deviate from the QED prediction by less than $1 \%$. Our measurements at energies between 14 and $46.78 \mathrm{GeV}$ agree well with this expectation, as shown in Table I. form

The angular distribution of $e^{+} e^{-} \rightarrow \mu^{+} \mu^{-}$has the

$$
\begin{aligned}
& d \sigma / d \cos \theta \\
& \quad=\left(\pi \alpha^{2} / 2 s\right)\left[R_{\mu \mu}\left(1+\cos ^{2} \theta\right)+B \cos \theta\right] .
\end{aligned}
$$

The factor

$$
B=-4 \chi g_{A}^{e} g_{A}^{\mu}+8 \chi^{2} g_{V}^{e} g_{V}^{\mu} g_{A}^{e} g_{A}^{\mu}
$$

TABLE I. Data sample, $R_{\mu \mu}, A_{\mu \mu}$, and the predicted values of the asymmetry, $A_{\mathrm{th}}$.

\begin{tabular}{lrrrrr}
\hline $\begin{array}{c}\sqrt{s} \\
(\mathrm{GeV})\end{array}$ & $\begin{array}{c}\int L d t \\
\left(\mathrm{pb}^{-1}\right)\end{array}$ & $\begin{array}{c}\mu \mu \\
(\text { No. })\end{array}$ & \multicolumn{1}{c}{$R_{\mu \mu}{ }^{\mathrm{a}}$} & \multicolumn{1}{c}{$\begin{array}{c}A_{\mu \mu} \\
(\%)\end{array}$} & \multicolumn{1}{c}{$\begin{array}{c}A_{\mathrm{th}} \mathrm{b}^{\mathrm{b}} \\
(\%)\end{array}$} \\
\hline 14.0 & 1.5 & 472 & $1.04 \pm 0.05$ & $+5.3 \pm 5.0$ & -1.5 \\
22.5 & 3.0 & 357 & $1.02 \pm 0.05$ & $-4.3 \pm 6.1$ & -3.5 \\
34.6 & 76.3 & 3658 & $0.98 \pm 0.016$ & $-11.7 \pm 1.7$ & -8.5 \\
39.8 & 9.3 & 341 & $1.01 \pm 0.05$ & $-10.9 \pm 5.7$ & -12.0 \\
44.6 & 23.5 & 673 & $0.99 \pm 0.04$ & $-16.7 \pm 4.0$ & -16.0 \\
\hline \hline
\end{tabular}

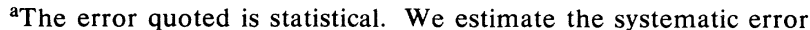
to be \pm 0.04 , mainly from the uncertainty in the luminosity.

${ }^{\mathrm{b}} m_{Z^{0}}=93 \mathrm{GeV}, \sin ^{2} \theta_{\mathrm{W}}=0.22$.

determines the forward-backward asymmetry which is given by

$$
A_{\mu \mu}=\frac{N\left(\theta<90^{\circ}\right)-N\left(\theta>90^{\circ}\right)}{N\left(\theta<90^{\circ}\right)+N\left(\theta>90^{\circ}\right)}=\frac{3}{8} \frac{B}{R_{\mu \mu}} .
$$

$N\left(\theta<90^{\circ}\right)$ is the number of events with the $\mu^{-}$in the forward hemisphere, and $N\left(\theta>90^{\circ}\right)$ the number in the backward hemisphere. The asymmetry, $A_{\mu \mu}$, depends upon the axial-vector couplings of the electron and the muon and is practically independent of the vector couplings,

$$
A_{\mu \mu}=-\frac{3}{2} \chi \frac{g_{A}^{e} g_{A}^{\mu}\left(1-2 \chi g_{V}^{e} g_{V}^{\mu}\right)}{\left(1-2 \chi g_{V}^{e} g_{V}^{\mu}+\ldots\right)} \approx-\frac{3}{2} \chi g_{A}^{e} g_{A}^{\mu}=-\frac{3}{8} \frac{1}{\sin ^{2} \theta_{\mathrm{W}} \cos ^{2} \theta_{\mathrm{W}}} \frac{s}{m_{Z^{0}}^{2}-s} g_{A}^{e} g_{A}^{\mu}
$$

The asymmetry is negative at energies below the $Z^{0}$ mass only if $g_{A}^{e}$ and $g_{A}^{\mu}$ have the same sign.

QED alone produces a small positive forwardbackward asymmetry, through the interference between the one- and two-photon exchange processes and between the initial- and final-state bremsstrahlung. The QED asymmetry is calculated by a Monte Carlo program of Behrends and Kleiss. ${ }^{7}$ It is found to be $+1.4 \%$ for our detector and it is practically independent of energy. Theoretical calculations of the asymmetry, including the full electroweak radiative corrections, have been performed with different sets of renormalized physical parameters. ${ }^{8-11}$ These calculations agree at the full one-loop level. The size of the electroweak radiative corrections varies with the choice of renormalized physical parameters. In the scheme ${ }^{10}$ employing the fine-structure constant, $\alpha$, and the physical masses of the $W^{ \pm}$and the $Z^{0}$, the size of the correction is $+1.4 \%$ as previously obtained with pure QED. With use of this approach, the data then need be corrected only for QED radiative effects in order to be compared with the prediction of Eq. (10). Note that in this scheme the weak mixing angle $\theta_{\mathrm{W}}$ is defined by

$$
\cos \theta_{\mathrm{W}}=m_{\mathrm{W}} / m_{Z^{0}},
$$

and is not modified by radiative corrections. If we fit Eq. (7) to the corrected data for $\cos \theta<0.8$ and then extrapolate over the full angular range $(\cos \theta<1)$ we obtain the asymmetries at different energies as given in Table I and in Fig. 1. The errors shown are statistical. Systematic errors are less than $0.5 \% .^{2,3}$ The dashed curve in Fig. 1 is the standard-model prediction using $\sin ^{2} \theta_{\mathrm{W}}=0.22$ and $m_{Z^{0}}=93 \mathrm{GeV}$. The agreement between our measured data and the predictions of the standard electroweak model is good.

Using Eqs. (2)-(10), we determine the values of $\sin ^{2} \theta_{\mathrm{W}}$ and $m_{Z^{0}}$, which are shown as $68 \%$ and $95 \%$ confidence level (C.L.) contours in Fig. 2. As discussed above, these contours include electroweak radiative corrections. In order to test the standard model, we compare these contours to the values of $\sin ^{2} \theta_{\mathrm{w}}$ and $m_{Z^{0}}$, determined by Arnison et al. (UA1) ${ }^{12}$ and Bagnaia et al. (UA2) ${ }^{13}$ in experiments at CERN. Note that we use the measured masses, $m_{\mathrm{W}}$ and $m_{Z^{0}}$, from the UA1 and UA2 experiments, and Eq. (11) to determine their values of $\sin ^{2} \theta_{W}$. This procedure is consistent with the definition in Eq. (11) and our electroweak radiative corrections. ${ }^{14}$ This comparison (Fig. 


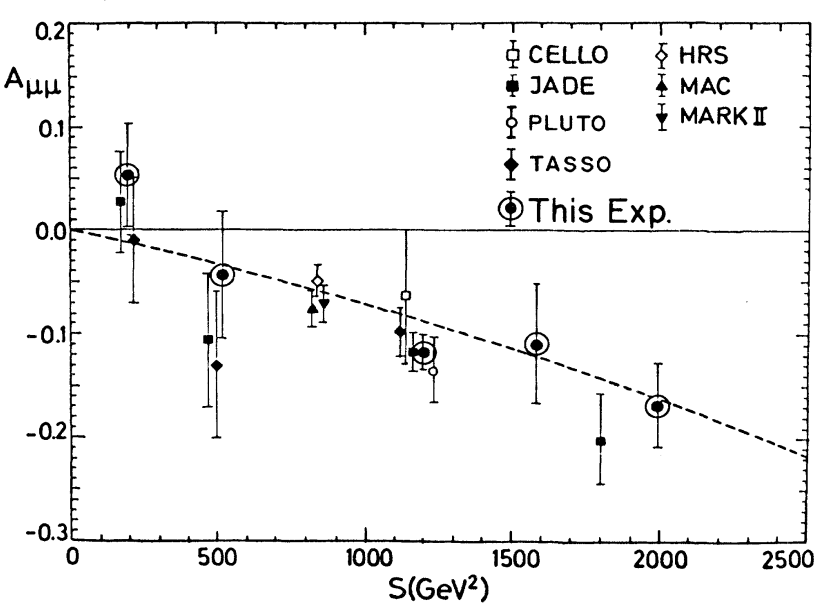

FIG. 1. $A_{\mu \mu}$ as a function of $s$. The dashed curve is the standard model prediction using $\sin ^{2} \theta_{\mathrm{W}}=0.22$ and $m_{Z^{0}}=93$ $\mathrm{GeV}$.

2) is a direct test of the standard model with a purely leptonic reaction. We find agreement within the errors. Taking the average value of $m_{Z^{0}}=93 \pm 2 \mathrm{GeV}$ from the CERN experiments, ${ }^{12,13}$ we find from our measurement

$$
\sin ^{2} \theta_{\mathrm{W}}=0.17_{-0.02}^{+0.03} \pm 0.01
$$

and at the $95 \%$ C.L.

$$
0.13<\sin ^{2} \theta_{\mathrm{W}}<0.25 \text {. }
$$

The first error is statistical, including the uncertainty in the $Z^{0}$ mass, the second is systematic. Combining all published PETRA and PEP results we obtain

$$
\left\langle\sin ^{2} \theta_{\mathrm{W}}\right\rangle=0.167_{-0.015}^{+0.020} \text {. }
$$

(See also Refs. 15 and 16.) Our result can be compared with that obtained from neutrino-electron and antineutrino-electron elastic scattering by Bergsma et al. ${ }^{17}$ and Ahrens et al. ${ }^{18}$ Their average value, uncorrected for radiative effects, ${ }^{19}$ is

$$
\sin ^{2} \theta_{\mathrm{W}}=0.212 \pm 0.023 \text {. }
$$

These are to be compared to the world average, ${ }^{20}$

$$
\left\langle\sin ^{2} \theta_{\mathrm{W}}\right\rangle=0.217 \pm 0.014,
$$

which is measured by lepton-nucleon and antileptonnucleon inelastic scattering and includes radiative corrections.

Alternatively, we can determine $m_{Z^{0}}$ from our data by using the world average for $\sin ^{2} \theta_{W}$. We find

$$
m_{Z^{0}}=87 \pm 5 \mathrm{GeV} \text {. }
$$

The errors are $68 \%$ C.L. measurement errors. Use of all $e^{+} e^{-}$data changes the central value to $86 \mathrm{GeV}$ and the error to \pm 3.3 . Taking $m_{Z^{0}}=93 \mathrm{GeV}$ and

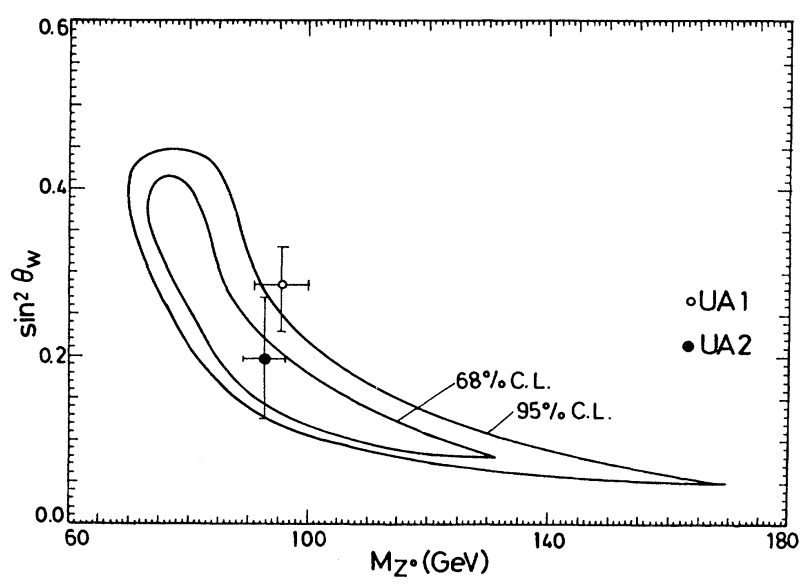

FIG. 2. Contours showing the correlation between $\sin ^{2} \theta_{\mathrm{W}}$ and $m_{Z^{0}}$. The points are measurements by Arnison et al. (UA1) and Bagnaia et al. (UA2); the error bars indicate the corresponding $68 \%$ C.L. contour value.

$\sin ^{2} \theta_{\mathrm{W}}=0.217$, we find from our data that $g_{A}^{e} g_{A}^{\mu}=0.30_{-0.04}^{+0.03} \pm 0.02$.

Our measurement of $R_{\mu \mu}$ and $A_{\mu \mu}$ also allows us to test models with extended gauge groups. In Ref. 3 we have described a test at $\langle\sqrt{s}=35 \mathrm{GeV}$ for $\mathrm{SU}(2) \otimes \mathrm{U}(1) \otimes G$ models. These models are described by the Hamiltonian for neutral currents

$$
H_{\mathrm{NC}}=\left(4 G_{\mathrm{F}} / \sqrt{2}\right)\left[\left(j_{\mu}^{(3)}-\sin ^{2} \theta_{\mathrm{W}} j_{\mu}^{e m}\right)^{2}+C\left(j_{\mu}^{e m}\right)^{2}\right] .
$$

$C$ is a constant which depends upon the group $G, j_{\mu}^{(3)}$ is the third component of the weak isospin current, and $G_{\mathrm{F}}$ is the Fermi weak-coupling constant. Our new data at higher average energy, $\langle\sqrt{s}\rangle=44 \mathrm{GeV}$, determine the parameter $C<0.035$, at $95 \%$ C.L. We have also tested the left-right symmetric model with a $\mathrm{SU}(2)_{L} \otimes \mathrm{SU}(2)_{R} \otimes \mathrm{U}(1)$ gauge group. $^{21}$ This model has two neutral gauge bosons and it is described by a Langrangean of the type

$$
L=g J_{L} W_{L}+g J_{R} W_{R}+g_{Y} J^{Y} B^{Y} .
$$

$g_{Y}$ is the hypercharge coupling constant. The symmetry breaking is arranged in the Higgs sector such that $m_{W_{L}}<<m_{W_{R}}$ and only left-handed currents contribute at low energies. Using the formalism given in Ref. 21, we find for the masses of the neutral bosons

$$
m_{Z_{2}}>150 \mathrm{GeV} \text { with } 95 \% \text { C.L., }
$$

with

$$
m_{Z_{1}}=85-100 \mathrm{GeV}
$$

In summary: (1) Our measurements of the muon charge asymmetry and the total cross section in the reaction $e^{+} e^{-} \rightarrow \mu^{+} \mu^{-}$support the standard elec- 
troweak theory. (2) We obtain $\sin ^{2} \theta_{\mathrm{W}}=0.17 \pm 0.03$ \pm 0.01 for $m_{Z^{0}}=93 \pm 2 \mathrm{GeV}$ and $m_{Z^{0}}=87 \pm 4 \mathrm{GeV}$ for $\sin ^{2} \theta_{\mathrm{W}}=0.217 \pm 0.014$ from a purely leptonic reaction. (3) In a left-right symmetric model with the $\mathrm{SU}(2)_{L} \otimes \mathrm{SU}(2)_{R} \otimes \mathrm{U}(1)$ extended gauge group, we find the mass of $m_{Z_{2}}>150 \mathrm{GeV}$ with $95 \%$ C.L. with $m_{Z_{1}}=85-100 \mathrm{GeV}$.

The DESY management has given the Mark J Collaboration continuing and generous encouragement. We are very appreciative of the excellence of the PETRA machine group and its support. We acknowledge profitable discussions with L. M. Seghal and T. G. Rizzo.

III. Physikalisches Institut, Technische Hochschule, Aachen is supported by the Deutsches Bundministerium fuer Forschung und Technologie. Two of us (M.H. and K.N.) acknowledge support from the Pakistan Atomic Energy Commission.

${ }^{1}$ S. L. Glashow, Nucl. Phys. 22, 579 (1961); S. Weinberg, Phys. Rev. Lett. 19, 1264 (1967), and Phys. Rev. D 5, 1412 (1972); A. Salam, in Elementary Particle Theory, edited by N. Svartholm, Nobel Symposium No. 8 (Wiley, New York, 1969), p. 361; S. L. Glashow et al., Phys. Rev. D 2, 1285 (1970).

2B. Adeva et al., Phys. Rev. Lett. 48, 1701 (1982).

${ }^{3}$ B. Adeva et al., Phys. Rep. 109, Nos. 3\&4, 133 (1984).

${ }^{4} \mathrm{H}$. J. Behrend et al. (CELLO Collaboration), Z. Phys. C 14, 283 (1982); W. Bartel et al. (JADE Collaboration), Phys. Lett. 108B, 140 (1982), and Z. Phys. C 26, 507 (1985); Ch. Berger et al. (PLUTO Collaboration), Z. Phys. C 7, 289 (1981), and Z. Phys. C 21, 53 (1983); M. Althoff et al. (TASSO Collaboration), Z. Phys. C 22, 13 (1984); R. Brandelik et al., Phys. Lett. 110B, 173 (1982).

${ }^{5}$ E. Fernandez et al. (MAC Collaboration), Phys. Rev. Lett. 50, 1238 (1983); T. Himel et al. (MARK I Collaboration), Phys. Rev. Lett. 41, 449 (1978); M. E. Levi et al. (MARK II Collaboration), Phys. Rev. Lett. 51, 1941 (1984); M. Derrick et al. (HRS Collaboration), Argonne National Laboratory Report No. HEP-PR-84-71 (unpublished); D. Bender et al., Phys. Rev. D 30, 515 (1984).

${ }^{6}$ R. Budny, Phys. Lett. 55B, 227 (1975).

${ }^{7}$ F. A. Behrends and R. Kleiss, Nucl. Phys. B177, 237 (1981), and Nucl. Phys. B186, 22 (1981), and programs which they have supplied

${ }^{8} \mathrm{G}$. Passarino and M. Veltman, Nucl. Phys. B160, 151 (1979); G. Passarino, Nucl. Phys. B204, 237 (1982).

${ }^{9}$ W. Wetzel, Nucl. Phys. B227, 1 (1983); B. W. Lynn and R. G. Stuart, Nucl. Phys. B253, 216 (1985).

${ }^{10}$ M. Böhm and W. Hollik, Nucl. Phys. B204, 45 (1982), and Phys. Lett. 139B, 213 (1984).

${ }^{11}$ F. A. Behrends, R. Kleiss, and S. Jadach, Nucl. Phys. B202, 63 (1983); R. Decker, E. A. Paschos, and R. W. Brown, Phys. Rev. Lett. 52, 1192 (1984).

${ }^{12} \mathrm{G}$. Arnison et al. (UA1 Collaboration), Phys. Lett. 129B, 273 (1983), and Phys. Lett. 126B, 398 (1983).

${ }^{13}$ P. Bagnaia et al. (UA2 Collaboration), Phys. Lett. 129B, 130 (1983), and Z. Phys. C 24, 1 (1984). Note that the UA2 point in Fig. 2 has error bars deduced from a correlated $68 \%$ C.L. contour.

${ }^{14}$ More precise values of $\sin ^{2} \theta_{\mathrm{W}}$ are obtained from the measurements of $m_{W}$ and the value of the muon decay constant $G_{\mu}$ including radiative corrections. See Refs. 12 and 13.

${ }^{15} \mathrm{~A}$ combined fit of all $e^{+} e^{-}$data are found in $\mathrm{R}$. Prepost, in Proceedings of the Twenty-Second International Conference on High Energy Physics, edited by A. Meyer and E. Wieczore (Akademie der Wissenschaftendor DDR, Zeuth, East Germany, 1984), p. 227; C. K. Bowdery, ibid., p. 229; A. Böhm, in Proceedings of the Nineteenth Recontre de Moriond on Electroweak Interactions and Unified Theories, La Plagne, 1984 (to be published).

16W. Bartel et al. (JADE Collaboration), Z. Phys. C 26, 507 (1985); Ch. Berger et al. (PLUTO Collaboration), DESY Report No. 84-121, 1984 (unpublished).

${ }^{17} \mathrm{~F}$. Bergsma et al. (CHARM Collaboration), Phys. Lett. 117B, 272 (1982).

${ }^{18}$ L. A. Ahrens et al. (Brookhaven Neutrino Collaboration), Phys. Rev. Lett. 54, 18 (1984).

${ }^{19}$ The electroweak corrections are negligible in comparison to the error. See S. Sarantakos, A. Sirlin, and W. J. Marciano, Nucl. Phys. B217, 84 (1983).

${ }^{20}$ A. Sirlin and W. J. Marciano, Nucl. Phys. B189, 442 (1981); C. L. Llewellyn-Smith and J. Wheater, Phys. Lett. 105B, 486 (1981); W. J. Marciano, in Proceedings of the International Symposium on Lepton and Photon Interaction at High Energies, Ithaca, New York, 1983, edited by D. J. Cassel and D. L. Kreinick (Newman Laboratory of Nuclear Studies, Cornell University, Ithaca, N.Y., 1983), p. 80.

${ }^{21}$ T. G. Rizzo and G. Senjanović, Phys. Rev. D 24, 704 (1981); V. Barger et al., Phys. Rev. D 28, 1618 (1983); L. M. Sehgal, III. Physikalisches Institut, Technische Hochschule, Aachen Report No. PITHA 84/03, 1984 (to be published). 